\title{
Preface
}

\section{Unintentional injury after traumatic brain injury: Issues, assessment, and reducing risk}

\author{
Juliet Haarbauer-Krupa* \\ Division of Unintentional Injury Prevention, National Center for Injury Prevention and Control, \\ Centers for Disease Control and Prevention, Atlanta, GA, USA \\ Tel.: +1 770488 4142; E-mail: JHaarbauerKrupa@cdc.gov.
}

Unintentional poisonings, falls, and motor vehicle crashes contribute to reduced life expectancy among persons with TBI [1]. Research examining adolescents and adults with moderate to severe TBI who participated in rehabilitation showed that they were twice as likely to die from an unintentional injury that occurs following their TBI compared to individuals in the rehabilitation population of similar age, sex and race. In particular, fall related injuries are both a leading cause of an initial TBI and more likely to lead to death following a TBI [1]. Persistent health problems among those with a TBI contribute to limitations in function, disability and reduced life quality [2-4], all of which can lead to increased injury risk. The public health burden of TBI extends beyond primary prevention to research and programs addressing how best to reduce injuries after experiencing a TBI. This special issue is dedicated to work on unintentional injury after a TBI with a focus on understanding injury risk related to changes in cognition, health, and behavior as a result of the TBI.

Dr. Grant Baldwin and colleagues provide a commentary to describe the public health burden of TBI and current efforts to reduce unintentional injuries, from a sports or recreation-related injury, a motor vehicle crash, or an older adult fall. The commentary offers insights into the public health approach to injury prevention and how these efforts can reduce injuries after TBI.
Dr. Carlson and colleagues report on the associations of TBI and motor vehicle crash (MVC)-related hospitalization of service members who use Veteran's Health Administration (VA) Healthcare. This study reveals a substantial risk for post-TBI MVC in veterans with a TBI. The authors suggest that driver safety interventions may reduce the risk of subsequent TBI after the initial injury.

Dr. Seel and colleagues characterize the development and validation of a measure for assessing safety and risk at home and in the community for individuals with moderate to severe TBI following hospital discharge. The emphasis of this article is on the theoretical support for their conceptual framework that identifies individual level factors that underlie safety risk that leads to unintentional injuries. The authors propose to examine safety in terms of "patient-centered" outcome measures that evaluate health, function and participation as part of daily experiences.

Dr. Kolakowsky-Hayner describes the effects of a TBI on functional skills and aligns these with risk for unintentional injury. Following a TBI, changes in cognitive and health functioning can result in reduction in an individual's' ability to monitor their environment and safety skills that can create an increased risk for unintentional injury. Plans that includes healthcare provider consideration of health monitoring, informing individuals with TBI about the 
importance of making behavioral choices to minimize injury risk and education of caregivers about recognizing unsafe environments and monitoring of family members are suggested practices for prevention of unintentional injuries after TBI.

Dr. Colontonio and colleagues examine return to work (RTW) for individuals who sustained a workplace TBI. The authors describe factors that align with (RTW) using a mixed methods (qualitative and quantitative) approach. Demographic factors, effects from the TBI, employer factors and family support are aspects contributing to understanding reduction of injury risk at work and RTW status.

Sustaining a TBI can lead to increased risk for unintentional injuries. Efforts to better understand modifiable risk factors and to design and evaluate effective interventions to prevent unintentional injuries after TBI may reduce the healthcare burdens and costs as well as improve rehabilitation outcomes.

\section{References}

[1] Harrison-Felix, C., Pretz, C., Hammond, F. M., Cuthbert, J. P., Bell, J., Corrigan, J., Miller, A. C., \& Haarbauer-Krupa, J. (2014). Life expectancy after inpatient rehabilitation for Traumatic Brain Injury in the United States. Journal of Head Trauma Rehabilitation, 31, 1-9.

[2] Masel, B. E., \& DeWitt, D. S. (2010). Traumatic Brain Injury: A disease process, not an event. Journal of Neurotrauma, 27, 1529-1540.

[3] Riggio, S., \& Wong, M., (2009). Neurobehavioral sequelae of Traumatic Brain Injury, Mount Sinai Journal of Medicine, 76, 163-172.

[4] Walker, W. C., \& Pickett, T. C. (2007). Motor impairment after severe traumatic brain injury: A longitudinal multicenter study. Journal of Rehabilitation Research \& Development, 44(7), 975-982. 